\title{
Tick Borne Disease Induced Pancytopenia
}

\author{
Mina Said ${ }^{\mathbf{1}}$, Ekta Tirthani ${ }^{\mathbf{1}}$ id \\ 1 Rochester General Hospital, NY, USA
}

\begin{abstract}
One of the uncommon and often missed causes of acute pancytopenia is tick-borne illnesses, mainly Human Monocytic Ehrlichiosis (HME) / Human Granulocytic Anaplasmosis (HGA) and Babesiosis, though the specific mechanism of the former is still unknown. The challenges in such diagnosis are that the tests for many tick-borne diseases have poor validity, and also that these diseases can co-exist, making the exact etiology of the hematopoietic defect more difficult to identify. This report demonstrates a case of acute pancytopenia secondary to tick-borne illness, which is a rare presentation. This was based on the exposure history, exclusion of other causes, Erythema Migrans lesion, response to doxycycline, and the known limitations of testing.

A 60-year-old Caucasian female with Rheumatoid arthritis, presented in Rochester, New York during summer, with a six-day history of vague symptoms including fatigue, headache, poor appetite, and nausea. She was frequently visiting her husband who lives in a cabin in a near wooded area. Records showed she had a history of Lyme disease that got successfully treated one year prior, but did not recall any tick bites lately. She was found to have fever, acute pancytopenia, and transaminitis. Her initial labs also showed high LDH and CRP. Otherwise, Reticulocytes, Vitamin B12, Folate, Lactic acid, have all been normal, as well as her imaging including chest X-ray, head CT, and abdominal ultrasound. A bone marrow aspirate showed nonspecific reactive changes. Infectious diseases team later noted a circular skin lesion with pale center suggesting Erythema Migrans, with a thorough workup only positive for Lyme IgG and EBV IgG, but negative for Legionella antigen, Leptospirosis antibody, HIV, Parvovirus B19, Hepatitis panel, HME/HGA testing, blood smear, and blood cultures. She was started on a Doxycycline trial on Day 4 for probable tick-borne illness with rapid improvement in her symptoms and lab results.
\end{abstract}

Keywords: Tick, Pancytopenia

\section{INTRODUCTION}

$\mathrm{P}$ ancytopenia has a wide differential diagnosis. One of the uncommon and often missed causes is tick-borne illnesses, mainly Human Monocytic Ehrlichiosis (HME) $\perp$ / Human Granulocytic Anaplasmosis (HGA) and Babesiosis, though the specific mechanism of the former is still unknown $(1,2)$. Noteworthy to mention is that the tests for tick-borne diseases so far have poor validity, and also that these diseases can co-exist such as Lyme disease, HGA and Babesiosis, making the exact etiology of the hematopoietic defect more difficult to identify.
Corresponding Author: Mina Said

E-mail:

minasem2005@yahoo.com

Received: February 20, 2021

Accepted: April 24, 2021

Published: April 30, 2021

Suggested citation: Said M, Tirthani E. Tick Borne Disease Induced Pancytopenia Infect Dis Clin Microbiol 2021; 1: 35-38.

DOI: $10.36519 / \mathrm{idcm} .2021 .32$ 


\section{THE CASE:}

A 60-year-old female with a medical history of Systemic Lupus (SLE) and Rheumatoid Arthritis (on Hydroxychloroquine), presented in summer at Rochester, NY with a 6-day history of fatigue, headache, generalized body aches, and poor appetite, associated with a 2-day history of fever, abdominal pain, nausea, and urinary frequency. She denied any chest pain, dyspnea, cough, upper respiratory symptoms, dysuria, rashes, bleeding, or dizziness. She had history of Lyme disease one year prior to presentation and was treated with Doxycycline. She has no known allergies and takes no other medications. She quit smoking recently, drinks alcohol occasionally, and uses no drugs. She has recently been going to visit her husband who lives in a cabin in a wooded area at Hammondsport, NY, but does not recall any tick bites. She has one pet cat at home. She has no recent sick contacts. She was in South Carolina the month prior, with no other travel history.

Day 1: Physical exam showed a temperature of 38.4 $\mathrm{C}$ and mild tenderness of the right upper abdominal quadrant. Initial workup showed white blood count (WBC) 1.4 (from a 4.7 baseline) with bandemia \& reactive lymphocytes, platelets 16 (from baseline 200), hemoglobin (Hb) 11 (from baseline 15.5) with normal MCV and reticulocytes, aspartate transaminase (AST) 250, alanine transaminase (ALT) 97, alkaline phosphatase (ALP) 117, vitamin B12 >2000, folate 21, lactate 1.1, Chest X-ray \& Head CT were unremarkable. Home medications were held, and IV fluids were given.

Day 2: Physical exam showed temperature of 39.2 C, pallor, small left axillary lymph nodes. complete blood count $(\mathrm{CBC})$ and comprehensive metabolic panel (CMP) were roughly the same, lactate dehydrogenase (LDH) 697, Haptoglobin 184, C-reactive protein (CRP) 157, D-dimer > 7650, fibrinogen 319, negative urinalysis, negative Monospot, hepatitis panel only positive for total Hepatitis A virus antibodies (later repeated negative), abdominal ultrasound was unremarkable. She received 2 units of platelets with an expected response. She then underwent a bone marrow aspirate, which only showed nonspecific reactive changes with later

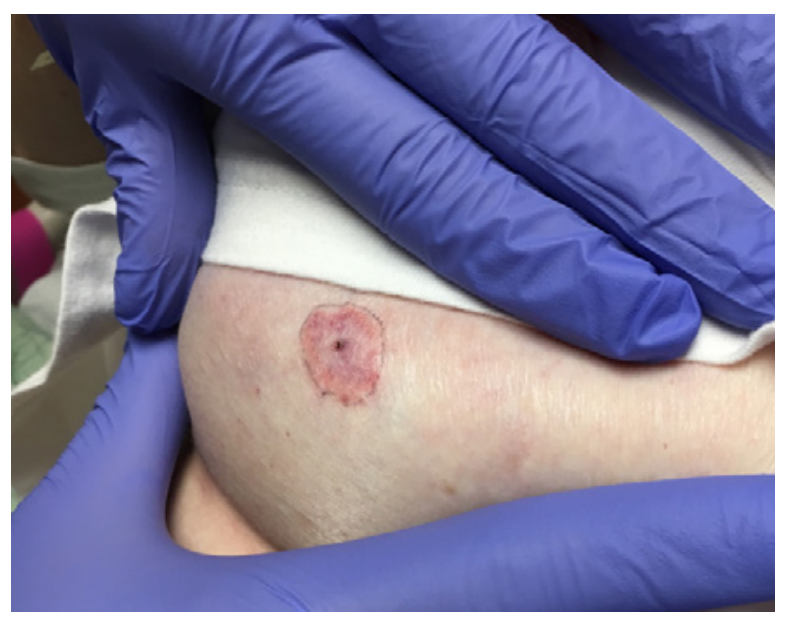

Figure 1. Erythema after tick bite.

negative flow cytometry for malignancy. Also, the Infectious diseases team was consulted, who asked for more workup that follows.

Day 3: Her temperature normalized, but still felt fatigued. WBC up to 3.0, $\mathrm{Hb}$ 11.4, Platelets up to 23, AST 189, ALT 78, ALP 128, HIV negative, Legionella Antigen negative, Lyme disease antibodies were positive for IgG at level 2.34 (from 8.7 one year earlier).

Day 4: Physical Exam however noted a right breast circular $2 \mathrm{~cm}$ erythematous skin lesion with a pale center suggesting Erythema Migrans (EM). WBC 5.1, $\mathrm{Hb}$ 10.7, Platelets 28 with noted samples clumping affecting the count, EBV and CMV positive for IgG only, Leptospirosis antibody negative, Blood cultures remained negative, Ehrlichiosis antibody negative. Then she was started empirically on a 10-day trial course of Doxycycline for possible convalescent or early false negative Ehrlichiosis or Anaplasmosis.

Day 5-6: WBC up to 7.1, Platelets estimated to be 100 due to clumping, AST 529, ALT 306, ALP 125, Parvovirus negative, Anaplasmosis antibody negative.

Day 7: Liver function tests came down to AST 372, ALT 337, and ALP 130. She was discharged to complete the Doxycycline course and restarted her home medications. Her labs completely normalized in one week. 


\section{DISCUSSION}

Our patient presented with acute pancytopenia, where the differentials include Bone marrow failure secondary to either aplastic anemia or myelodysplastic syndrome or infections like HIV, EBV, CMV or some drugs / irradiation side effects, Bone marrow infiltration, Megaloblastic anemia, Paroxysmal nocturnal hemoglobinuria, Hepatosplenomegaly, SLE and Disseminated Intravascular coagulopathy (DIC). The bone marrow aspirate showed nonspecific reactive changes with no massive hypo-cellularity or evidence of infiltration, making Aplastic anemia and marrow invasion less likely. Megaloblastic anemia was also excluded by normal MCV, folate and B12 levels. She had no hepatosplenomegaly on abdominal ultrasound. D-dimer was high, but Fibrinogen \& coagulation studies did not match DIC. She did have a positive ANA history but no other criteria to suggest SLE flare as a cause, especially with normal reticulocytes and indirect bilirubin.

Possible infections were tested, including EBV, CMV, HIV, Parvovirus B19 and Hepatitis viruses which were all unremarkable. Legionella antigen and Leptospirosis antibody were also tested, given the associated transaminitis and were negative as well. Her tick-borne illnesses testing showed negative antibodies for Ehrlichiosis \& Anaplasmosis, no morulae on peripheral blood smear, while Lyme disease IgG was positive, though unsurprising given her past infection. However, given the history of prior tickborne disease, and woods exposure in northeastern USA summer, the EM lesion on exam, the typical picture of cytopenias and high liver function tests, low sensitivity of the HME/HGA tests especially early in the course, as well as the good response to Doxycycline trial, the main diagnosis for our case is Tick-borne disease induced.

Lyme disease very rarely causes pancytopenia independently, though is reported to be associated with it in the setting of co-infection with Babesiosis or HGA or both, especially that all three can be transmitted through the same tick, Ixodes Scapularis (3). The HME/HGA testing includes peripheral smear (first week, 3-20\% sensitivity), PCR (first week, 55$87 \%$ sensitivity), IFA (second week, 94-100\% sensitivity) (4). These antibody testing can also cross react with Lyme disease antibodies positivity (2). Various cytopenias have been reported with HME/ HGA since their discovery in 1986 and 1994 respectively, with pancytopenia being the rarest noted (4). The mechanism by which tick-borne diseases cause pancytopenia is still unclear, with some suggestions pointing towards HLH and myelosuppressive chemokines. $(1,7,9)$

\section{CONCLUSION}

This report demonstrates a case of pancytopenia secondary to tick-borne illness, which highlights one atypical presentation of these diseases. Therefore, it is better to always keep it in mind for the differential diagnosis of acute pancytopenia. It also goes in-depth with the diagnostic modalities of tick-borne diseases and their limitations so far in challenging scenarios.
Informed Consent: Written consent was obtained from the patients.

Peer-review: Externally peer-reviewed

Author Contributions: Concept - ALL.; Design - ALL.; Supervision - ALL; Data Collection and/or Processing - ALL; Analysis and/or Interpretation -ALL; Literature Review - ALL; Writer - ALL; Critical Reviews - ALL.
Conflict of Interest: The authors have no conflict of interest to declare.

Financial Disclosure: The authors declared that this study has received no financial support. 


\section{REFERENCES}

1 Borjesson D, Macnamara K, Johns J, Winslow G. Anaplasma phagocytophilum and Ehrlichia muris induce cytopenias and global defects in hematopoiesis. Clin Microbiol Infect. 2009;15 Suppl 2(Suppl 2):66-67. doi: 10.1111/j.1469-0691.2008.02182.x

2 Cunha BA, Chandrankunnel JG, Hage JE. Ehrlichia chaffeensis human monocytic ehrlichiosis with pancytopenia. Scand J Infect Dis. 2012;44(6):473-474. doi:10.3109/00365548.2011.633550

3 Mehrzad R, Bravoco J. Pancytopenia in Lyme disease. BMj Case Rep. 2014;2014:bcr2013201079. Published 2014 Mar 4. doi: 10.1136/bcr-2013-201079

4 Hilal T, Snapp WK. The perils of country life: human monocytic ehrlichiosis. Am J Med. 2015;128(8):831-833. doi: 10.1016/j. amimed.2015.04.003

5 Hay CM, Shepard JA, Hyle EP, Duncan LM. Case records of the Massachusetts General Hospital. Case 23-2014. A 41-yearold man with fevers, rash, pancytopenia, and abnormal liver function. N Engl J Med. 2014;371(4):358-366. doi: 10.1056/NEIMcpc1404140
6 Lee J, Azzawi S, Peluso MJ, Richterman A, Batlle HR, Yialamas MA. Smear Campaign: Misattribution of Pancytopenia to a Tick-Borne Illness. J Gen Intern Med. 2018;33(4):570-572. doi: 10.1007/s11606-017-4241-5

7 Marko D, Perry AM, Ponnampalam A, Nasr MR. Cytopenias and clonal expansion of gamma/delta T-cells in a patient with anaplasmosis: a potential diagnostic pitfall. J Clin Exp Hematop. 2017;56(3):160-164. doi: 10.3960/jslrt.56.160

8 Sykes DB, Zhang EW, Karp Leaf RS, Nardi V, Turbett SE. Case 10-2020: An 83-Year-Old Man with Pancytopenia and Acute Renal Failure. N Engl J Med. 2020;382(13):1258-1266. doi: 10.1056/ NEJMcpc1916250

9 Abbott KC, Vukelja SJ, Smith CE, et al. Hemophagocytic syndrome: a cause of pancytopenia in human ehrlichiosis. Am J Hematol. 1991;38(3):230-234. doi: 10.1002/ajh.2830380315

10 Allen MB, Pritt BS, Sloan LM, et al. First reported case of Ehrlichia ewingii involving human bone marrow. J Clin Microbiol. 2014;52(11):4102-4104. doi: 10.1128/JCM.01670-14 